\title{
A Smartphone App Is Feasible for Outpatient Cirrhotic Ascites Management
}

Patricia Bloom, MD; Madeline Marx, MD; Thomas Wang, MD; Ashwini Arvind; Jasmine Ha, MBBS; Bradley Green, MBBS; James Richter, MD

Gastroenterology, Massachusetts General Hospital, Boston, MA, United States

Corresponding Author:

Patricia Bloom, MD

Massachusetts General Hospital

Gastroenterology

55 Fruit $\mathrm{St}$

Boston, MA

United States

Phone: 9784600538

Email: ppbloom@partners.org

\begin{abstract}
Background: Ascites, or accumulation of abdominal free fluid, develops in two-thirds of patients with cirrhosis. Ascites is painful and, if inadequately managed, can lead to life-threatening complications, including spontaneous bacterial peritonitis and kidney failure. Body weight is an effective proxy for ascites volume; therefore, monitoring daily weights is recommended for optimal ascites management. At present, patients with ascites rarely proactively alert providers of significant weight gains, and there are no widely available technologies specifically designed for ascites monitoring.
\end{abstract}

Objective: The objective of this pilot study is to assess the feasibility of a smartphone app to manage outpatient ascites.

Methods: In this feasibility study, cirrhotic patients with significant ascites requiring specialist management are identified through an inpatient hepatology consult census and outpatient referrals. Each candidate is sent home with a Bluetooth-connected scale, which transmits weight data to the PGHD Connect Smartphone App, and then via the cloud into the electronic medical record (EMR). Weights are monitored every weekday by study staff and alerts are sent to providers if their patients' weight changes by $\geq 5 \mathrm{lbs}$ within a week or from the weight documented at discharge. The primary outcomes are percentage of study enrollment days when weight data was successfully transmitted into the EMR and percentage of weight alerts to which providers responded.

Results: Seventy-eight cirrhotic patients were identified as requiring active management of ascites. Of these patients, 8 did not own a smartphone, 23 were encephalopathic, and thus were excluded; another 1 declined to participate, and 3 were consented but subsequently withdrawn due to physical limitation or death prior to hospital discharge. Each patient is enrolled in the program for 28 days. Of the 16 patients currently enrolled, 5 (31\%) are male, mean age is 60.9 years (SD 11.1), $13(81 \%)$ were enrolled as inpatients, $8(50 \%)$ have non-alcoholic steatohepatitis cirrhosis, 4 (25\%) alcohol-associated cirrhosis, and 2 (12.5\%) viral cirrhosis. At this interim analysis, transmission of weight data into the EMR has successfully occurred on $70 \%$ of study enrollment days. Patients experienced technology issues during $10 \%$ of days enrolled. Of the total 20 weight alerts to date, 12 (60\%) were triggered by weight loss $\geq 5 \mathrm{lb}$ in one week, 7 (35\%) by weight gain $\geq 5 \mathrm{lb}$ in one week, and $1(5 \%)$ by weight gain $\geq 5 \mathrm{lb}$ since discharge. Providers responded to $13(65 \%)$ of the weight alerts within 24 hours. Of the 13 alerts with a provider response, 7 $(54 \%)$ were followed by a call or email to the patient to discuss care, $4(31 \%)$ a scheduled appointment, $4(31 \%)$ a change in diuretic dosage, $3(23 \%)$ scheduling for paracentesis (procedure to remove ascites fluid), and 3 (23\%) further laboratory workup. To date, there have been 13 readmissions.

Conclusions: On the basis of our interim analysis, we demonstrate feasibility of a martphone app to facilitate ascites management. We report encouraging rates of patient and provider engagement. This innovation shows promise in enabling early intervention and enhancing quality of life in cirrhotic patients. Future studies will investigate the efficacy of mobile health technology to improve outcomes in this population.

(iproc 2019;5(1):e15130) doi: $10.2196 / 15130$ 
Edited by R Palacholla; this is a non-peer-reviewed article. Submitted 21.06.19; accepted 14.08.19; published 02.10.19.

Please cite as:

Bloom P, Marx M, Wang T, Arvind A, Ha J, Green B, Richter J

A Smartphone App Is Feasible for Outpatient Cirrhotic Ascites Management

iproc 2019;5(1):e15130

URL: http://www.iproc.org/2019/1/e15130/

doi: $\underline{10.2196 / 15130}$

PMID:

(CPatricia Bloom, Madeline Marx, Thomas Wang, Ashwini Arvind, Jasmine Ha, Bradley Green, James Richter. Originally published in Iproceedings (http://www.iproc.org), 02.10.2019 This is an open-access article distributed under the terms of the Creative Commons Attribution License (https://creativecommons.org/licenses/by/4.0/), which permits unrestricted use, distribution, and reproduction in any medium, provided the original work, first published in Iproceedings, is properly cited. The complete bibliographic information, a link to the original publication on http:/www.iproc.org/, as well as this copyright and license information must be included. 